\title{
Magnetohydrodynamic Jeffrey nanoliquid flow with thermally radiative Newtonian heat and mass species
}

\author{
Sabir Ali Shehzad \\ Department of Mathematics, COMSATS University Islamabad, Sahiwal 57000, Pakistan. \\ e-mail: sabirali@ciitsahiwal.edu.pk
}

Received 8 May 2018; accepted 11 July 2018

\begin{abstract}
This study characterizes the properties of Newtonian heat and mass species conditions in three-dimensional Jeffrey nanoliquid flow generated by the movement of thermally radiative surface. The liquid flow is electrically conducting through the consideration of magnetic field. The aspects of heat absorption, generation, and thermal radiation are considered in the equation of energy conservation. The boundary layer phenomenon is employed to obtain the mathematical expressions of considered physical model. These equations are solved via homotopic scheme. The convergence of homotopic solutions is validated by the numerical data. The importance of physical constraints on temperature, and nanoparticle concentration of liquid is visualized by the graphical results.
\end{abstract}

Keywords: Three-dimensional flow; Jeffrey liquid; nanoparticles; Newtonian conditions; bidirectional surface.

PACS: 65.80.-g; 82.60.Qr

\section{Introduction}

Complex liquids have common nature of viscoelasticity. Some examples of complex viscoelastic liquids include suspensions of colloidal, polymer solutions, and polymer melts. Such fluids are generally involved in blow, and injection molding, extrusions, lab on a chip, inkjet printers, and many others. Viscoelastic behavior of liquids may affect the different aspects of flows like transport, and energy dissipation, hydraulic resistance, flow stability and mixing performance [1]. Intra and inter molecules reactions of polymer chains may generate the viscoelasticity. Analysis and modeling of viscoelastic fluid flows have technical and scientific interests. Researchers of modern world have paid full attention to elaborate the mechanism of viscoelastic materials for better quality in industrial manufacturing of products. Many complex rheological expressions of viscoelastic materials had been developed in the past. A very fabulous model of viscoelastic liquids is known as Jeffrey fluid. This model has the features of stress retardation, and relaxation. Various investigators have adopted this model to discuss the different problems of fluid flows under multiple situations. For example, Turkyilmazoglu and Pop [2] modeled the flow of Jeffrey fluid due to shrinking or stretching of surface and express the exact behavior of govern mathematical expressions. Das et al. [3] disclosed the nature of thermal magnetic field in stretched Jeffrey liquid flow with transport of melting heat. Gravitational aspects on Jeffrey liquid flow with solutal and thermal stratification have been reported by Abbasi et al. [4]. Hayat et al. [5] addressed the convective heating effects in hydromagnetic stagnant flow of Jeffrey fluid. Qayyum et al. [6] discussed the reactive Jeffrey fluid flow over variable thickness surface.

Nanofluids have achieved special attention due to their broad applications in modern science, and technology like glass fiber, electronic chips, micro-computers, wire draw- ing, renewable energy sources, engine oils, vehicles, systems of thermal energy storage, and many others. Some common base liquids such as oil, grease, water, and ethylene glycol are poor conductors and have lesser thermal efficiency. Researchers have developed modern technique to augment the thermal performance of such liquids with insertion of nanoparticles. The involvement of solid particles may alter the density, viscosity, thermal conductivity, and heat capacity of such liquids. In nanofluids mechanisms, there are various slip factors include thermophoretic, and Brownian movement aspects which are responsible to motion of nanoparticles in base liquids. Concentration of nanoparticles may not be constant due to presence of velocity slip factor of nanoliquids. Investigators of the modern era have proven that nanoparticles have an ability of transport energy, and also alter the thermophysical features. Buongiorno [7] was the first who explored the forces which have impacts on nanoparticles velocity. He reported that Brownian movement and thermophoretic are the forces which generate drift-flux in nanoparticles. After that, different attempts have been made by using the Buongiorno model to explore the mechanism of such factors under various circumstances and conditions [8-20].

Here our attention is to discuss the nature of Newtonian heat and mass transportation in three-dimensional magnetohydrodynamic flow of Jeffrey nanoliquid induced by the bidirectional movement of surface. In the past, the investigators discuss the various fluid flows problems by only considering the Newtonian heating aspects. They ignored the Newtonian mass transport effects. In practical situations, heat and mass transport occur simultaneously and if Newtonian heat phenomenon takes place, then the importance of Newtonian mass cannot be ignored. Here we considered both Newtonian heat and mass conditions because the problems of modern industrial processes. This phenomenon is more realistic, and practical. We also considered the solar radiation term in 


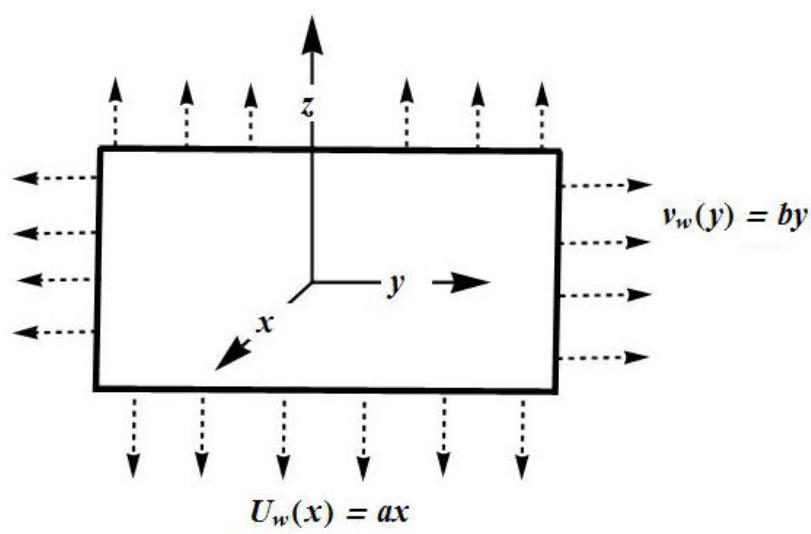

FIGURE 1. Geometry of problem.

energy expression. All the governing equations are tackled by homotopic procedure [21-26]. The results are plotted for different values of physical constraints.

\section{Governing problems}

We assumed the three-dimensional steady-state flow of Jeffrey liquid induced by the movement of thermally radiative surface (Fig. 1). The impacts of heat absorption, generation, and thermal radiation are accounted in the expressions of energy. The Newtonian heat and mass species conditions are imposed at boundary of surface. The equations of considered flow phenomenon through boundary layer theory are

$$
\begin{aligned}
\frac{\partial u}{\partial x}+ & \frac{\partial v}{\partial y}+\frac{\partial w}{\partial z}=0 \\
u \frac{\partial u}{\partial x}+ & =\frac{\partial u}{\partial y}+w \frac{\partial w}{\partial z}=\frac{v}{1+\delta_{1}}\left(\frac{\partial^{2} u}{\partial z^{2}}+\delta_{2}\right. \\
& \times\left(\frac{\partial u}{\partial z} \frac{\partial^{2} v}{\partial x \partial z}+\frac{\partial v}{\partial z} \frac{\partial^{2} v}{\partial y \partial z}+\frac{\partial w}{\partial z} \frac{\partial^{2} v}{\partial z^{2}}\right. \\
& \left.\left.+u \frac{\partial^{3} u}{\partial x \partial z^{2}}+v \frac{\partial^{3} u}{\partial y \partial z^{2}}+w \frac{\partial^{3} u}{\partial z^{3}}\right)\right)-\frac{\sigma B_{0}^{2}}{\rho_{f}} u \\
u \frac{\partial u}{\partial x} & +v \frac{\partial u}{\partial y}+w \frac{\partial w}{\partial z}=\frac{v}{1+\delta_{1}}\left(\frac{\partial^{2} v}{\partial z^{2}}+\delta_{2}\right. \\
& \times\left(\frac{\partial u}{\partial z} \frac{\partial^{2} v}{\partial x \partial z}+\frac{\partial v}{\partial z} \frac{\partial^{2} v}{\partial y \partial z}+\frac{\partial w}{\partial z} \frac{\partial^{2} v}{\partial z^{2}}\right. \\
& \left.\left.+u \frac{\partial^{3} u}{\partial x \partial z^{2}}+v \frac{\partial^{3} u}{\partial y \partial z^{2}}+w \frac{\partial^{3} u}{\partial z^{3}}\right)\right)-\frac{\sigma B_{0}^{2}}{\rho_{f}} v
\end{aligned}
$$

$$
\begin{aligned}
& u \frac{\partial T}{\partial x}+v \frac{\partial T}{\partial y}+w \frac{\partial T}{\partial z}=\frac{\partial^{2} T}{\partial z^{2}}+\tau\left(D_{B} \frac{\partial C}{\partial z} \frac{\partial T}{\partial z}\right. \\
&\left.+\frac{D_{T}}{T_{\infty}}\left(\frac{\partial T}{\partial z}\right)^{2}\right)+\frac{1}{(\rho c)_{f}} \frac{16 \sigma^{*} T_{\infty}^{3}}{3 k^{*}} \frac{\partial^{2} T}{\partial z^{2}} \\
&+Q\left(T-T_{\infty}\right), \\
& u \frac{\partial C}{\partial x}+v \frac{\partial C}{\partial y}+w \frac{\partial C}{\partial z}=D_{B} \frac{\partial^{2} C}{\partial z^{2}}+\frac{D_{T}}{T_{\infty}} \frac{\partial^{2} T}{\partial z^{2}}
\end{aligned}
$$

where the respective velocity components in the $x-, y-$ and $z$ - directions are denoted by $u, v$ and $w, \delta_{1}$ the ratio of relaxation to retardation times, $\delta_{2}$ the retardation time, $T$ the fluid temperature, $v=(\mu / \rho)$ the kinematic viscosity, $\mu$ the dynamic viscosity of fluid, $\rho_{f}$ the density of fluid, $\sigma$ the electrical conductivity, $\alpha$ the thermal diffusivity, $Q>0$ the heat generation parameter, and $Q<0$ represents the heat absorption parameter, $\tau=\left((\rho c)_{p} /(\rho c)_{f}\right)$ the ratio of nanoparticle heat capacity, and the base fluid heat capacity, $\sigma^{*}$ the Stefan-Boltzmann constant, $k^{*}$ the mean absorption coefficient, $D_{B}$ the Brownian diffusion coefficient, and $D_{T}$ the thermophoretic diffusion coefficient.

The boundary conditions can be written in the following forms:

$$
\begin{aligned}
& u=U_{w}(x)=a x, \quad v=V_{w}(y)=b y, \quad w=0 \\
& \frac{\partial T}{\partial z}=-h_{t} T, \quad \frac{\partial C}{\partial z}=-h_{c} C, \quad \text { at } \quad z=0 \\
& u \rightarrow 0, \quad v \rightarrow 0, \quad T \rightarrow T_{\infty}, \quad C \rightarrow C_{\infty} \\
& \quad \text { as } \quad z \rightarrow \infty
\end{aligned}
$$

where $a$ and $b$ are the constants of dimension time inverse, $h_{t}$ conjugate coefficient of heat transport, and $h_{c}$ conjugate coefficient of mass transport.

By employing the following variables

$$
\begin{aligned}
& u=d x f^{\prime}(\xi), \quad v=d y g^{\prime}(\xi), \quad w=-\sqrt{d v}\left(f^{\prime}(\xi)+g(\xi)\right), \\
& \xi=z \sqrt{\frac{d}{v}}, \quad \theta(\xi)=\frac{T-T_{\infty}}{T_{\infty}}, \quad \phi(\xi)=\frac{C-C_{\infty}}{C_{\infty}}
\end{aligned}
$$

Eq. (1) is satisfied automatically, and Eqs. (2)-(7) can be converted in the following forms:

$$
\begin{aligned}
f^{\prime \prime \prime} & +\left(1+\delta_{1}\right)\left((f+g) f^{\prime \prime}-f^{2}\right)+D e_{1}\left(f^{\prime \prime 2}\right. \\
& \left.-(f+g) f^{\prime \prime \prime \prime}-g^{\prime} f^{\prime \prime \prime}\right)-\left(1+\lambda_{1}\right) M f^{\prime}=0, \\
g^{\prime \prime \prime} & +\left(1+\delta_{1}\right)\left((f+g) g^{\prime \prime}-g^{2}\right)+D e_{1}\left(g^{\prime \prime 2}\right. \\
& \left.-(f+g) g^{\prime \prime \prime \prime}-f^{\prime} g^{\prime \prime \prime}\right)-\left(1+\lambda_{1}\right) M g^{\prime}=0, \\
(1+ & \left.\frac{4}{3} T_{R}\right) \theta^{\prime \prime}+\operatorname{Pr}(f+g) \theta^{\prime}+N_{B} \theta^{\prime} \varphi^{\prime} \\
& +N_{T} \theta^{2}+\operatorname{Pr} S \theta=0,
\end{aligned}
$$




$$
\begin{aligned}
\varphi^{\prime \prime} & +\operatorname{Pr} \operatorname{Le}(f+g) \varphi^{\prime}+\left(N_{T} / N_{B}\right) \theta^{\prime \prime}=0, \\
f & =0, g=0, f^{\prime}=1, g^{\prime}=\delta, \theta^{\prime}=-N_{H}(1-\theta(0)), \\
\phi^{\prime} & =-N_{C}(1-\phi(0)) \text { at } \eta=0, \\
f^{\prime} & \rightarrow 0, g^{\prime} \rightarrow 0, \theta \rightarrow 0, \phi \rightarrow 0 a s \eta \rightarrow \infty
\end{aligned}
$$

where $D e=\delta_{2} d$ is the Deborah number, $M_{g}=\left(\sigma B_{0}^{2} / d \rho_{f}\right)$ the magnetic parameter, $\alpha=b / a$ is a ratio parameter, $\operatorname{Pr}=$ $v / \alpha$ is the Prandtl number, $T_{R}=4 \sigma T_{\infty}^{3} / k k^{*}$ is the thermal radiation parameter, $S=Q / d \rho c_{p}$ is the internal heat generation parameter, $N_{B}=\left(\tau D_{B} / v\right)$ is the Brownian motion parameter, $N_{T}=\left(\tau D_{T} / v T_{\infty}\right)$ is the thermophoresis parameter, $N_{H}=h_{t} \sqrt{v / d}$ and $N_{C}=h_{C} \sqrt{v / d}$ are the Biot numbers, $L e=\alpha / D_{B}$ is the Lewis number, and prime shows the differentiation with respect to $\xi$. The local Nusselt number $N u \operatorname{Re}_{x}^{-1 / 2}$ and Sherwood number $S h \operatorname{Re}_{x}^{-1 / 2}$ in dimensionless forms can be described as follows:

$$
\begin{aligned}
N u \operatorname{Re}_{x}^{-1 / 2} & =N_{H}\left(1+\frac{4}{3} T_{R}\right)\left(1+\frac{1}{\theta(0)}\right), \\
S h \operatorname{Re}_{x}^{-1 / 2} & =N_{C}\left(1+\frac{1}{\varphi(0)}\right),
\end{aligned}
$$

in which $\operatorname{Re}_{x}=u_{w}(x) x / v$ is the local Reynolds number.

\section{Series solutions}

The initial guesses and auxiliary linear operators for present case can be selected in the forms:

$$
\begin{aligned}
f_{0}(\xi) & =1-\exp (-\xi), \quad g_{0}(\xi)=\delta(1-\exp (-\xi)), \\
\theta_{0}(\xi) & =\frac{N_{H} \exp (-\xi)}{1+N_{H}}, \quad \varphi(\xi)=\frac{N_{C} \exp (-\xi)}{1+N_{C}}, \\
L_{f} & =f^{\prime \prime \prime}-f^{\prime}, \quad L_{g}=g^{\prime \prime \prime}-g^{\prime} \\
L_{\theta} & =\theta^{\prime \prime \prime}-\theta, \quad L_{\varphi}=\varphi^{\prime \prime \prime}-\varphi .
\end{aligned}
$$

We observe that the auxiliary linear operators may satisfy the following expressions

$$
\begin{aligned}
L(f)\left(E_{1}+E_{2} e^{\xi}+E_{3} e^{-\xi}\right) & =0, \\
L(g)\left(E_{1}+E_{2} e^{\xi}+E_{3} e^{-\xi}\right) & =0, \\
L(\theta)\left(E_{7} e^{\xi}+E_{8} e^{-\xi}\right) & =0, \\
L(\varphi)\left(E_{9} e^{\xi}+E_{10} e^{-\xi}\right) & =0,
\end{aligned}
$$

where $E_{i}(i=1-10)$ are the arbitrary constants.

\section{Analysis of convergence and results}

An analytical technique namely homotopy analysis scheme has been implemented to obtain the solutions of governing mathematical expressions. It is a well-established fact that convergence of homotopic solutions greatly depends on the

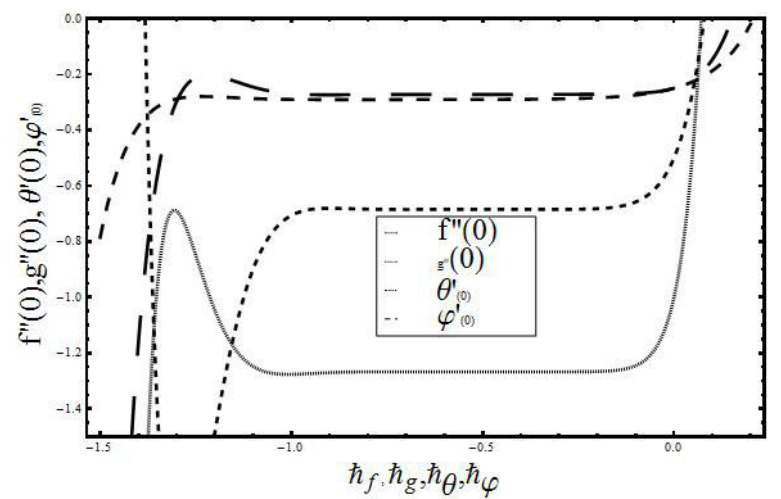

FIGURE 2. The $\hbar-$ curves for $f(\xi), g(\xi), \theta(\xi)$ and $\varphi(\xi)$ at 15 thorder of deformations when $D e=0.4, \delta_{1}=0.3, M_{g}=0.7$, $\operatorname{Pr}=1.5=L e, N_{B}=0.1=N_{T}, N_{H}=0.2=N_{C}, \delta=0.5$, $S=0.1$, and $T_{R}=0.3$.

TABLE I. Convergence of series solutions for different order of approximations when $D e=0.4, \delta_{1}=0.3, M_{g}=0.7, \operatorname{Pr}=1.5=$ Le, $N_{B}=0.1=N_{T}, N_{H}=0.2=N_{C}, S=0.1, T_{R}=0.3$, $\delta=0.5$, and $\hbar_{f}=\hbar_{g}=\hbar_{\theta}=\hbar_{\varphi}=-65 / 100$.

\begin{tabular}{ccccc}
\hline $\begin{array}{c}\text { Order of HAM } \\
\text { approximations }\end{array}$ & $-f^{\prime \prime}(0)$ & $-g^{\prime \prime}(0)$ & $-\theta^{\prime}(0)$ & $-\varphi^{\prime}(0)$ \\
\hline 01 & 1.26661 & 0.64143 & 0.26625 & 0.26777 \\
07 & 1.26718 & 0.68497 & 0.28911 & 0.27261 \\
12 & 1.26722 & 0.68482 & 0.29060 & 0.27274 \\
18 & 1.26727 & 0.68480 & 0.29080 & 0.27279 \\
23 & 1.26726 & 0.68489 & 0.29082 & 0.27278 \\
28 & 1.26732 & 0.68504 & 0.29081 & 0.27277 \\
35 & 1.26732 & 0.68504 & 0.29081 & 0.27277 \\
40 & 1.26732 & 0.68504 & 0.29081 & 0.27277 \\
\hline
\end{tabular}

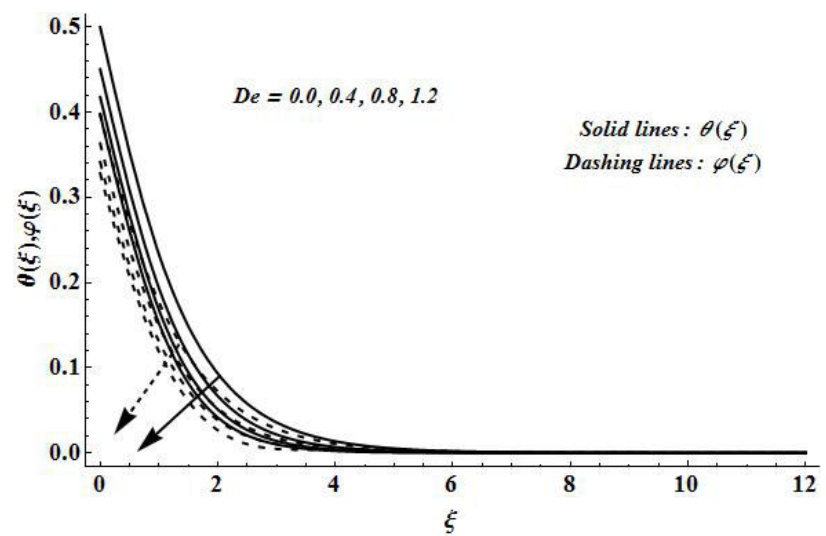

FIGURE 3. Curves of $\theta(\xi)$, and $\varphi(\xi)$ for different values of $D e$ when $\delta_{1}=0.3, M_{g}=0.7, \operatorname{Pr}=1.5=L e, N_{B}=0.1=N_{T}$, $N_{H}=0.2=N_{C}, \delta=0.5, S=0.1$, and $T_{R}=0.3$.

proper choice of auxiliary parameters. We plotted curves at $15^{\text {th }}$ order of deformations to select the reasonable values of auxiliary parameters $\hbar_{f}, \hbar_{g}, \hbar_{\theta}$ and $\hbar_{\varphi}$. For the present problem, the solutions should be convergent in the range of 


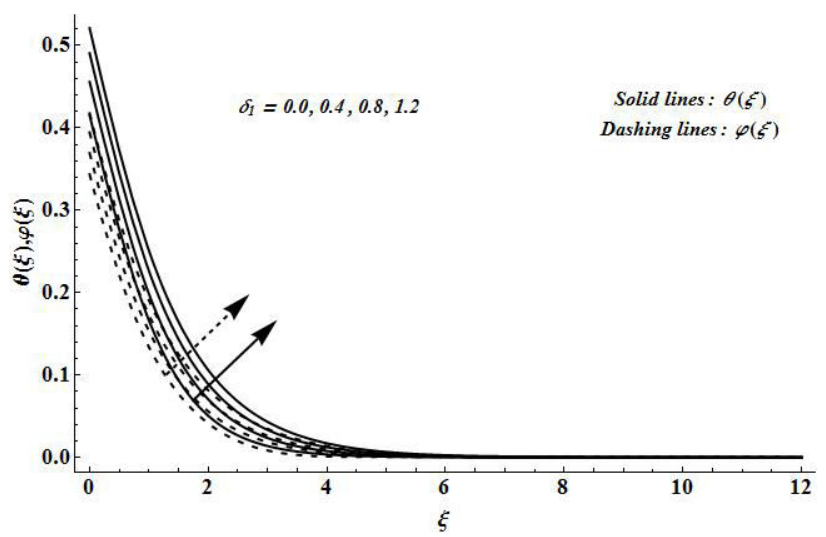

FIGURE 4. Curves of $\theta(\xi)$, and $\varphi(\xi)$ for different values of $\delta_{1}$ when $D e=0.4, M_{g}=0.7, \operatorname{Pr}=1.5=L e, N_{B}=0.1=N_{T}$, $N_{H}=0.2=N_{C}, \delta=0.5, S=0.1$, and $T_{R}=0.3$.

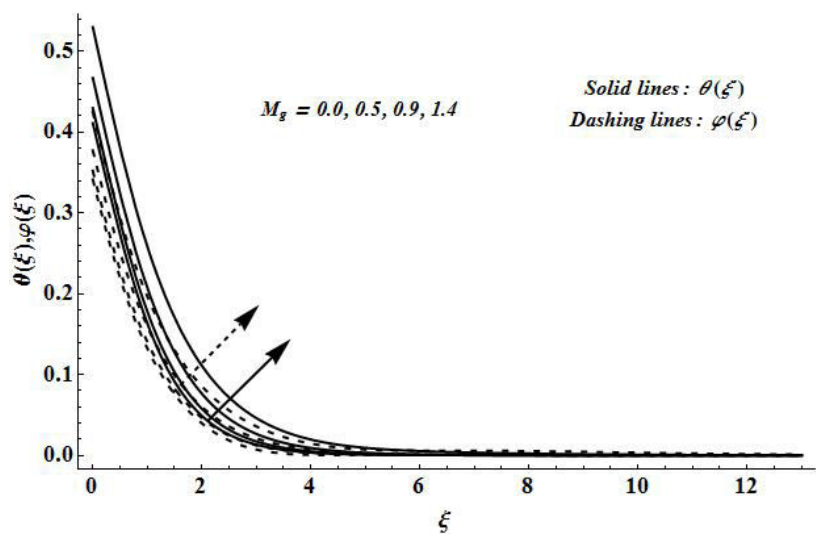

FIGURE 5. Curves of $\theta(\xi)$, and $\varphi(\xi)$ for different values of $M_{g}$ when $D e=0.4, \delta_{1}=0.3, \operatorname{Pr}=1.5=L e, N_{B}=0.1=N_{T}$, $N_{H}=0.2=N_{C}, \delta=0.5, S=0.1$, and $T_{R}=0.3$.

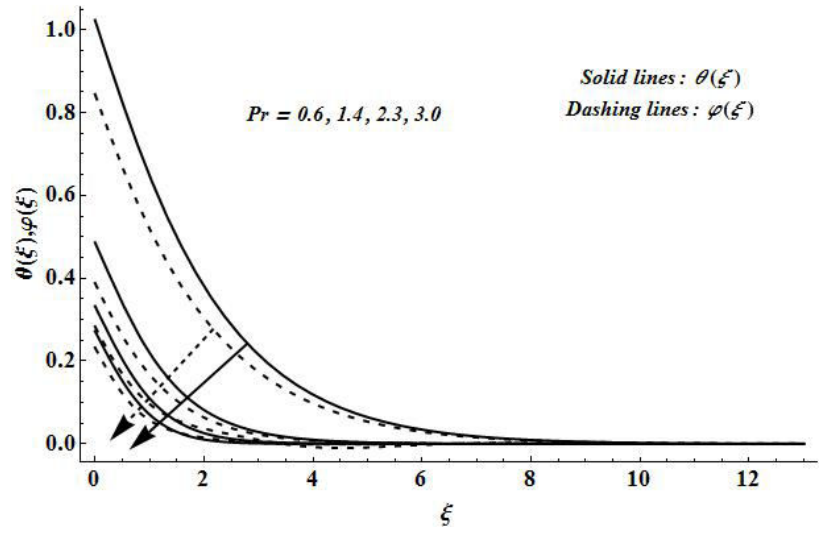

FIGURE 6. Curves of $\theta(\xi)$, and $\varphi(\xi)$ for different values of $\operatorname{Pr}$ when $D e=0.4, \delta_{1}=0.3, M g=0.7 \mathrm{Le}=1.5, N_{B}=0.1=N_{T}$, $N_{H}=0.2=N_{C}, \delta=0.5, S=0.1$, and $T_{R}=0.3$.

$-0.85 \leq \hbar_{f} \leq-0.15,-0.90 \leq \hbar_{g} \leq-0.20,-1.20 \leq$ $\hbar_{\theta} \leq-0.15$ and $-1.00 \leq \hbar_{\varphi} \leq-0.25$ (see Fig. 2). Table I executes that the present solutions converge from order of deformations. This Table is computed by choosing $\hbar_{f}=$ $\hbar_{g}=\hbar_{\theta}=\hbar_{\varphi}=-65 / 100, D e=0.4, \delta_{1}=0.3, M_{g}=0.7$, $\operatorname{Pr}=1.5=L e, \quad N_{B}=0.1=N_{T}, \quad N_{H}=0.2=N_{C}$,

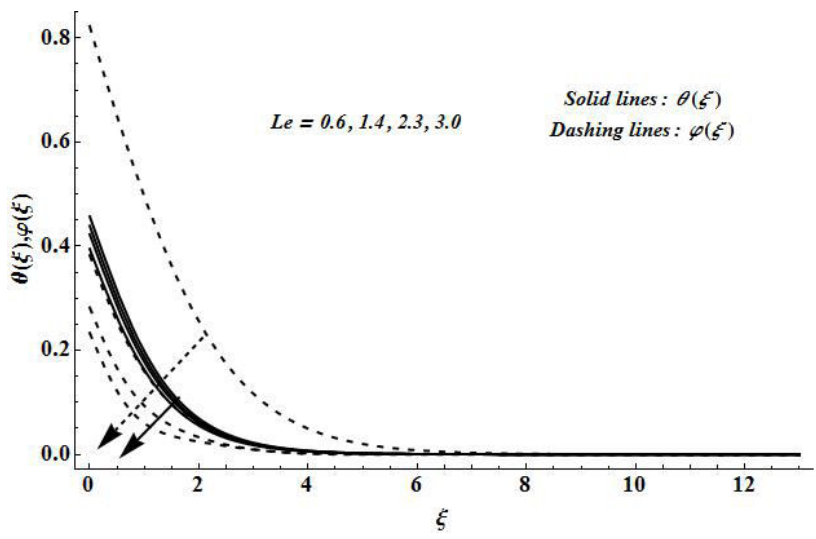

FIgURE 7. Curves of $\theta(\xi)$, and $\varphi(\xi)$ for different values of $L e$ when $D e=0.4, \delta_{1}=0.3, M g=0.7 \mathrm{Pr}=1.5 N_{B}=0.1=N_{T}$, $N_{H}=0.2=N_{C}, \delta=0.5, S=0.1$, and $T_{R}=0.3$.

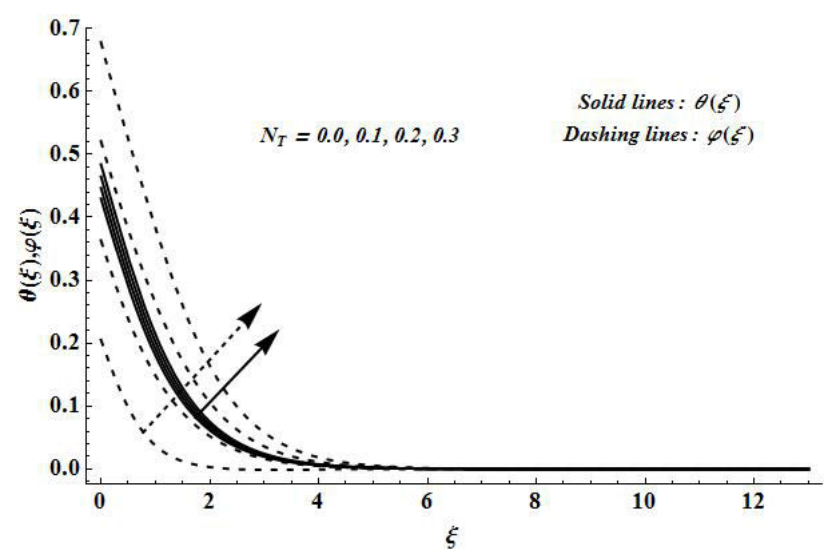

Figure 8. Curves of $\theta(\xi)$, and $\varphi(\xi)$ for different values of $N_{T}$ when $D e=0.4, \delta_{1}=0.3, M_{g}=0.7 \mathrm{Pr}=1.5=$ Le $N_{B}=0.1$, $N_{H}=0.2=N_{C}, \delta=0.5, S=0.1$, and $T_{R}=0.3$.

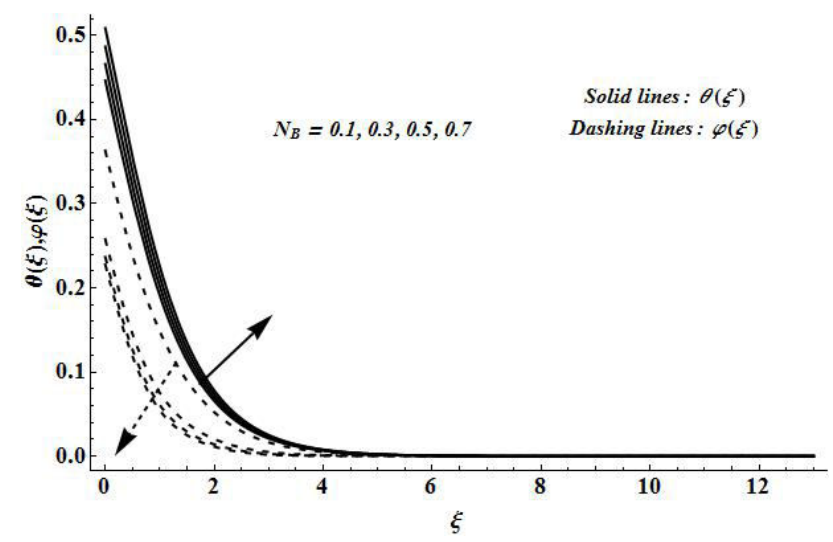

FIGURE 9. Curves of $\theta(\xi)$, and $\varphi(\xi)$ for different values of $N_{B}$ when $D e=0.4, \delta_{1}=0.3, M_{g}=0.7 \operatorname{Pr}=1.5=L e, N_{T}=0.1$, $N_{H}=0.2=N_{C}, \delta=0.5, S=0.1$, and $T_{R}=0.3$.

$S=0.1, T_{R}=0.3$, and $\delta=0.5$. Here we analyzed that the magnitude values of $f^{\prime \prime}(0)$ are larger in comparison to $g^{\prime \prime}(0)$, $\theta^{\prime}(0)$ and $\varphi(0)$.

Figures 3-10 are produced to elaborate the nature of emerging physical constraints on temperature $\theta(\xi)$ and nano- 


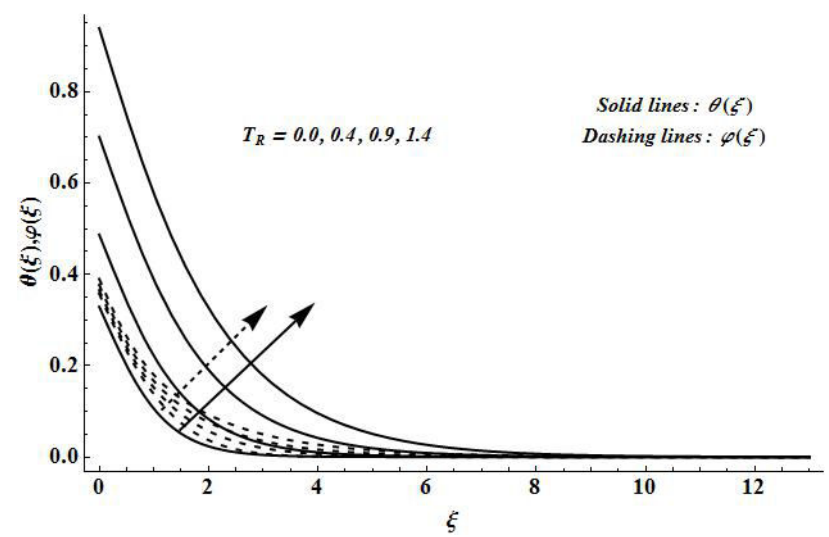

FIGURE 10. Curves of $\theta(\xi)$, and $\varphi(\xi)$ for different values of $T_{R}$ when $D e=0.4, \delta_{1}=0.3, M_{g}=0.7, \operatorname{Pr}=1.5=L e$, $N_{B}=0.1=N_{T}, N_{H}=0.2=N_{C}, \delta=0.5, S=0.1$.

particle concentration $\varphi(\xi)$. Figure 3 clearly describes that an increment in Deborah number $D e$ leads to smaller profiles of $\theta(\xi)$, and $\varphi(\xi)$ Here $D e$ directly depends on stress retardation $\delta_{2}$. The stress retardation is stronger with an enhancement in $D e$. Such stronger stress retardation creates a reduction in the profiles $\theta(\xi)$, and $\varphi(\xi)$. The results in the case of fluid liquid can be achieved when $D e=0$, and $\delta_{1}=0$. The features of ratio stress relaxation and stress retardation $\delta_{1}$ on $\theta(\xi)$ and $\varphi(\xi)$ are investigated in Fig. 4. The curves of $\theta(\xi)$ and $\varphi(\xi)$ are boost up when we use the larger $\delta_{1}$. From physical concept, the higher $\delta_{1}$ implies to larger stress relaxation in comparison to stress retardation. This fact leads to stronger temperature and nanoparticles concentration against the larger $\delta_{1}$. Figure 5 incorporates the impacts of magnetic parameter $M_{g}$ on $\theta(\xi)$, and $\varphi(\xi)$. This figure clearly executes that an increment in $M_{g}$ give rise to the profiles of $\theta(\xi)$ and $\varphi(\xi)$. Here the Lorentz force exists due to electrically conducting liquid. This Lorentz force becomes stronger for larger $M_{g}$ that resists the flow of liquid. Such resistance in liquid flow leads to higher temperature, and nanoparticle concentration. Further, the hydrodynamic flow situation can be created by setting $M_{g}=0$. In case of hydrodynamic flow, the profiles of $\theta(\xi)$, and $\varphi(\xi)$ are minimum position.

From Fig. 6, we evaluated the nature of Prandtl number $\operatorname{Pr}$ on $\theta(\xi)$, and $\varphi(\xi)$. The increasing values of $\operatorname{Pr}$ correspond to weaker profiles of temperature, and nanoparticle concentration. The conductivity of liquid is lesser for higher Pr, and stronger for smaller Pr due to fact the profiles of $\theta(\xi)$, and $\varphi(\xi)$ decay. The proper choice of $\operatorname{Pr}$ in manufacturing processes of industry is very essential because the suitable values of Pr are utilized to provide the best heat transport rate to achieve the highest quality product. The execution of Lewis number $L e$ on $\theta(\xi)$, and $\varphi(\xi)$ is presented in Fig. 7. This figure clearly elucidates that the variations in $\theta(\xi)$ are very lesser in comparison to $\varphi(\xi)$ as we use the larger Le. Moreover, the curves of $\theta(\xi)$, and $\varphi(\xi)$ are decreased for the increasing values of $L e$. The coefficient of Brownian diffusion becomes weaker for higher $L e$ because it is inversely related to it. Such weaker Brownian coefficient factor leads to decrement in the profiles of $\theta(\xi)$, and $\varphi(\xi)$.

The impact of parameter of thermophoretic $N_{t}$ on $\theta(\xi)$, and $\varphi(\xi)$ is discussed through Fig. 8. Figure 8 clearly depicts that profiles of both $\theta(\xi)$, and $\varphi(\xi)$ are risen due to rising values of $N_{T}$. The stronger $N_{T}$ corresponds to an enhancement in thermal conductivity of liquid due to consideration of nanoparticles. This higher thermal conductivity factor leads to higher $\theta(\xi)$, and $\varphi(\xi)$. The change in $\theta(\xi)$ for various values of $N_{T}$ are significant as compared to $\varphi(\xi)$. The increasing values of parameter of Brownian movement $N_{B}$ give risen to the profiles of $\theta(\xi)$, and $\varphi(\xi)$ (see Fig. 9). The liquid temperature $\theta(\xi)$ is boost up by an increment in $N_{B}$ while the curves of $\varphi(\xi)$ are retarded. In fact, $N_{B}$ is appeared in form of $\left(1 / N_{B}\right)$ that corresponds to weaker nanoparticle concentration. The nature of parameter of thermal radiation $T_{R}$ on liquid temperature, and nanoparticle concentration is described in Fig. 10. Here we investigated that the presence of $T_{R}$ give risen to $\theta(\xi)$, and $\varphi(\xi)$. The case of absence of radiative effect can be discussed when $T_{R}=0$. The radiation factor gives more heat to liquid due to which temperature and concentration are enhanced.

\section{Conclusions}

This research work executes the features of Newtonian conditions in three-dimensional Jeffrey liquid flow under the impact of magnetic field, thermal radiation, and suspension of nanoparticles. We employed the boundary layer concept to govern the expressions of physical phenomenon. The convergent solutions are developed and plotted. We observed that the ratio of stress relaxation and stress retardation $\delta_{1}$, and Deborah number $D e$ have totally reverse influences on liquid temperature $\theta(\xi)$, and nanoparticle concentration $\phi(\xi)$. An increment in magnetic parameter $M_{g}$ give risen to the profiles of $\theta(\xi)$, and $\varphi(\xi)$. This phenomenon occurs due to involvement of Lorentz force. The change in profiles of $\theta(\xi)$ for various $\mathrm{Pr}$ is significant as compared to profile of temperature in case of various $L e$. The liquid temperature, and nanoparticle concentration enhanced by increasing the values of thermophoretic factor $N_{T}$. An increase in factor of Brownian movement lead to higher temperature curves but lower curves of nanoparticle concentration. The involvement of radiation factor gradually enhanced the temperature of liquid. 
1. R. Comminal, J.H. Hattel, M.A. Alves, and J. Spangenberg, Journal of Non-Newtonian Fluid Mechanics 237 (2016) 1-15.

2. M. Turkyilmazoglu, and I. Pop, International Journal of Heat and Mass Transfer 57 (2013) 82-88.

3. K. Das, N. Acharya, and P.K. Kundu, Alexandria Engineering Journal 54 (2015) 815-821.

4. F.M. Abbasi, S.A. Shehzad, T. Hayat, and M.S. Alhuthali, Journal of Hydrodynamics Series B 28 (2016) 840-849.

5. T. Hayat, S. Asad, M. Mustafa, and A. Alsaedi, Computers and Fluids 108 (2015) 179-185.

6. Qayyum, S. Hayat, T. Alsaedi, A. and Ahmad, B. International Journal of Mechanical Sciences 134 (2017) 306-314.

7. J. Buongiorno, Journal of Heat Transfer-Transactions of ASME 128 (2006) 240-250.

8. M. Turkyilmazoglu, European Journal of Mechanics-B/Fluids 53 (2015) 272-277.

9. J. Li, L. Liu, L. Zheng, and B. Bin-Mohsin, Journal of the Taiwan Institute of Chemical Engineers 67 (2016) 226-234

10. M. Sheikholeslami, D.D. Ganji, and M.M. Rashidi, Journal of Magnetism and Magnetic Materials 416 (2016) 164-173.

11. B. Li, W. Zhang, L. Zhu, and L. Zheng, Powder Technology 310 (2017) 351-358

12. A. Rauf, S.A. Shehzad, T. Mushtaq, and T. Hayat, Journal of Nanofluids 6 (2017) 1134-1142.

13. K. Hsiao, Energy 130 (2017) 486-499.

14. M.I. Khan, A. Alsaedi, S.A. Shehzad, and T. Hayat, Results in Physics 7 (2017) 2255-2260.
15. M. Sheikholeslami, A.J. Chamkha, P. Rana, and R. Moradi, Chinese Journal of Physics 55 (2017) 2356-2370.

16. K. Hsiao, International Journal of Heat and Mass Transfer 112 (2017) 983-990.

17. T. Hayat, S. Qayyum, S.A. Shehzad, and A. Alsaedi, Journal of Molecular Liquids 230 (2017) 641-651.

18. F.A. Soomro, R. Haq, Z.H. Khan, and Q. Zhang, Chinese Journal of Physics 55 (2017) 1561-1568

19. X. Xiong, S. Chen, and B. Yang, Applied Mathematics and Mechanics 38 (2017) 585-602.

20. Nagendramma, V. Raju, C.S.K. Mallikarjun, B. Shehzad, S.A. and Leelarathnam, A. Applied Mathematics and MechanicsEnglish (Edition 2018) in press.

21. S.J. Liao, Homotopy analysis method in nonlinear differential equations. Springer \& Higher Education Press, (Heidelberg 2012).

22. M. Turkyilmazoglu, Communications in Nonlinear Science and Numerical Simulation 17 (2012) 4097-4103.

23. S. Han, L. Zheng, C. Li, and X. Zhang, Applied Mathematics Letters 38 (2014) 87-93

24. S.A. Shehzad, F.M. Abbasi, T. Hayat, and B. Ahmad, Applied Mathematics and Mechanics-English Edition 37 (2016) 761768.

25. M.A. Meraj, S.A. Shehzad, T. Hayat, F.M. Abbasi, and A. Alsaedi, Applied Mathematics and Mechanics 38 (2017) 557-566

26. M. Waqas, T. Hayat, S.A. Shehzad, and A. Alseadi, Physica B: Condensed Matter 529 (2018) 33-40 